\title{
Dewey: El significado democrático de la primacía de los hábitos
}

\section{Dewey: The democratic meaning of the priority of habits}

\author{
CARLOS MOUGAN*
}

\begin{abstract}
Resumen: La relevancia que Dewey concede a los hábitos en la deliberación sirve de clave para precisar la manera en que entendió la democracia. Aunque es posible encuadrarlo dentro de los defensores de la democracia deliberativa, la importancia que da a los factores no estrictamente epistémicos hace que rechace los supuestos intelectualistas que suelen caracterizar aquellas posiciones. La consecuencia es una teoría política normativa que entiende que la democracia requiere no sólo el cultivo de las capacidades cognitivas, sino el desarrollo de un ethos que haga posible la extensión de los valores democráticos. Palabras clave: Dewey, hábitos, democracia, deliberación, virtudes cívicas.
\end{abstract}

\begin{abstract}
The relevance Dewey bestows to habits in deliberation provides the key to clarify how he understood democracy. Although it is possible to list him among the advocates of deliberative democracy, the importance that he gave to non- epistemic factors led him to reject the intellectualist assumptions, that often characterize that position. The result is a normative political theory where it is understood democracy requires not only the growing cognitive abilities, but also the development of an ethos that fosters the extension of democratic values.
\end{abstract}

Keywords: Dewey, habits, democracy, deliberation, civic virtues.

John Dewey hizo de la prioridad de la acción sobre el pensamiento, de los hábitos sobre la conciencia, una de las claves alrededor de la cual gira su obra. Aclarar las implicaciones políticas y democráticas de dicha afirmación será el objeto de este trabajo. Se empezará por poner de manifiesto (1) en qué sentido los hábitos son importantes para el proceso de deliberación, lo que incluye precisar (2) que los hábitos son más que un instrumento, un elemento integral del conocimiento. Una de las claves de la relevancia ético-política de los hábitos radica en que (3) incorpora tanto elementos subjetivos como del medio desdibu-

Fecha de recepción: 19/11/2014. Fecha de aceptación: 24/03/2015.

* J. Carlos Mougan Rivero es Doctor en Filosofía y profesor titular de Filosofía moral y política en la Universidad de Cádiz. Correo electrónico: carlos.mougan@uca.es. Especialista en el pragmatismo americano, en especial en la obra de J. Dewey, sus investigaciones giran en torno a "calidad de la democracia y virtudes cívicas", "educación para la ciudadanía", "perfeccionismo liberal" y "ética profesional". Recientemente ha publicado "En defensa del perfeccionismo democrático" en Perfeccionismo. Entre la ética política y la autonomía personal, Pérez Chico y García Ruiz (eds.), 2014, Zaragoza, Prensas de la Universidad de Zaragoza, y, "An Engaged Fallibilistic Pluralism” en Confines of Democracy. Essays on the Philosophy of Richard J. Bernstein, Ramón del Castillo, Ángel M. Faerna \& Larry A. Hickman (eds.), (2015) Amsterdam \& New York, Rodopi. 
jando las tradicionales fronteras entre lo interno y lo externo, lo individual y lo social, lo ético y lo político, lo personal y lo institucional (4). Lo distintivo del análisis de Dewey de los hábitos (5) es que son estos los que abren una dimensión normativa que permite juzgar actitudes e instituciones democráticas. Por último, este análisis de los hábitos deja claro (6) que Dewey rechazó tanto el intelectualismo democrático que entiende la democracia como un triunfo de la razón, como de quienes rechazan toda pretensión epistémica de la misma. La importancia que Dewey concede a los hábitos en la deliberación se traduce en una teoría política que consiste en una propuesta normativa sobre la educación moral del ciudadano.

\section{La relevancia de los hábitos para la deliberación}

Tanto la formación de ideas como su ejecución dependen del hábito. Si pudiéramos concebir una idea correcta sin un hábito correcto, tal vez podríamos prescindir de éste para ejecutarla; pero un deseo toma forma definida sólo cuando está conectado con una idea, y ésta a su vez la toma sólo cuando hay un hábito que la respalde. Un hombre sólo se da cuenta de lo que es adoptar una postura correcta cuando de antemano ha podido ejecutar el acto de mantenerse erguido y sólo entonces puede invocar la idea requerida para la debida ejecución de ese acto. La acción debe anteceder al pensamiento y el hábito a la capacidad de evocarlo a voluntad. (Dewey, 1964, 39) ${ }^{1}$.

En este texto, como en general a largo de Naturaleza humana y conducta, Dewey señala que los humanos sabemos antes con los hábitos que con la conciencia. Esto sugiere, como el propio Dewey indica, una inversión de la manera usual en que interpretamos la relación entre hábito e ideas. La comprensión de la relevancia de los elementos no estrictamente cognitivos e inferenciales en la deliberación exige poner de manifiesto cómo se hace presente a lo largo del proceso que singulariza a la inteligencia y la investigación. Lo está, para empezar, en el origen mismo de la deliberación, pues esta comienza por la obstrucción del flujo continuo de la acción que caracteriza al hábito. Sólo cuando ocurre una interrupción de la acción, un conflicto entre hábitos, o entre un hábito y un impulso, tiene origen la deliberación. Son, por tanto, elementos no cognitivos o lógicos los que marcan el comienzo de la actividad intelectual. Lo que se requiere, de entrada, es una sensibilidad, una disposición que nos permita captar o ver el desajuste, la falta de adecuación de la situación. El pensamiento no comienza su tarea si no se ha sentido con antelación la existencia de un problema. Un hábito adormecedor, una sensibilidad abotargada, pueden hacer difícil o imposible la percepción de un obstáculo o una dificultad.

Hábito y sensibilidad no sólo aparecen como desencadenantes de la deliberación sino que también se hacen presentes en su desarrollo. Dewey define la deliberación "como un ensayo

1 En los casos en los que hay traducción castellana del libro de Dewey, las citas harán referencia a ella en relación con el año de su publicación. La demás referencias a los escritos de John Dewey se basarán en la edición crítica de las obras completas publicada por Southern Illinois University: EW (The Early Works), MW (The Middle Works) y LW (The Later Works). Las citas se harán según el modelo normalizado entre los estudiosos de la obra de Dewey: la inicial de las series son seguidas por el volumen y el número de la página. 
teatral (imaginario) de diversas posibles líneas de acción que están en competencia" (Dewey, 1964, 78). De este modo, la imaginación juega un papel central representando mentalmente distintas posibilidades y alternativas. De acuerdo con Fesmire "la deliberación moral es fundamentalmente imaginativa y adopta la forma de un ensayo dramático" (Fesmire, 2003, 4). Pero este juego imaginativo de diversos cursos de acción no es producto de una mente desencarnada o aislada de cualquier medio. Los diversos cursos posibles de acción están marcados por la existencia previa de la acción en la que el individuo está inmerso y que viene marcada por las pautas establecidas por los hábitos. Incluso si actúa la imaginación introduciendo novedades en el curso de acción que el agente se representa en la mente, esta modificación está hecha sobre la base de pautas de acción previas. La deliberación está condicionada, incluso en su aspecto de creación de novedades, por el hábito del que emergen. Como señala Alexander "la imaginación emerge como la necesidad del hábito de expandirse e integrarse con el cuerpo total del comportamiento" (Alexander, 1993, 386). Pero, más aún, en ese ensayo teatral imaginario son los elementos no inferenciales los que servirán finalmente para evaluar los distintos cursos de acción y, por consiguiente, los que determinarán la decisión a tomar. En esto, la deliberación está guiada por el mismo carácter objetivo y experiencial que siguen los cursos de acción. Así, "tanto en el pensamiento como en la acción manifiesta, los objetos experimentados al seguir un curso de acción atraen, repelen, satisfacen, molestan, activan y retardan”. (Dewey, 1964, 180).

Por su parte, el resultado de la deliberación supone la armonización de las distintas preferencias que entran en conflicto. La solución tiene que atender a integrar las exigencias de los impulsos y los requerimientos de los hábitos. Por tanto, habrá de ser no una solución que rompa con los elementos anteriores, impulsos, hábitos, deseos, etc., sino una que logre integrarlos en una respuesta que resulte satisfactoria desde el punto de vista de la situación. "La elección es razonable cuando nos induce a actuar con sensatez, es decir, con consideración a los derechos de cada uno de los hábitos e impulsos antagónicos”. (Dewey, 1964, 182).

Por consiguiente, el análisis de la deliberación supone la mediación entre los componentes emotivos y racionales siendo así que la inteligencia consiste finalmente en la armonización de nuestros deseos y comportamientos con los de los demás. Son exitosas aquellas deliberaciones que restauran el equilibrio con el medio y con los demás. El propio Dewey dice claramente que el objetivo de la deliberación es "reanudar la continuidad, recobrar la armonía, utilizar los impulsos sueltos y dar una nueva dirección del hábito" (Dewey, 1964, 186). Así pues, se produce una inversión de la tradición racionalista de pensamiento. En ella los hábitos y las emociones aparecen como elementos distorsionantes del buen juicio, aquel que no se deja llevar por elementos ajenos al devenir de la dinámica racional. Por el contrario, para Dewey no se trata de oponer el pensamiento contra los hábitos sino de modificar los ya existentes para hacerlos más adecuados al medio, para que den respuesta a las circunstancias problemáticas que provocan la interrupción del hábito y lograr, finalmente, un mayor grado de integración y armonía. Lo que necesitamos, como señala Pappas, para "contrarrestar la seducción de imágenes y apelaciones emocionales que distorsionan la investigación es más, no menos, hábitos emocionales e imaginativos" (Pappas, 2008, 253-254). Dewey lo afirmó explícitamente: "la conclusión no es que la fase emocional y apasionada de la acción pueda o deba ser eliminada a beneficio de una razón fría e impasible. Más pasiones, no menos es lo que se necesita" (Dewey, 1964, 
183) Al final del ejercicio de la deliberación, de la tarea del pensamiento, de nuevo nos encontramos con los hábitos. Es ahí en su renovación y adecuación donde hallamos el índice de que el pensamiento ha llevado a cabo su tarea.

\section{El valor epistémico de los hábitos}

Aun cuando, tal y como se ha mostrado, los hábitos tienen una fuerte presencia en todo el proceso de deliberación queda preguntarnos si tienen o no un valor epistémico. Tomando como referencia el texto inicial la cuestión es ¿por qué sólo el que ha adoptado la posición correcta puede pensar correctamente sobre su significado? La pregunta es hasta qué punto, o en qué medida, el juicio correcto depende del hábito adquirido. Porque podría ser que el hábito fuera importante para que se emitiera un juicio correcto al poner las condiciones que hacen posible que el agente lo emita, pero que, al mismo tiempo, estas condiciones no tuvieran una incidencia en el contenido mismo. Hábito y juicio práctico quedarían, si este fuera el caso, en una relación meramente externa, siendo los primeros un elemento auxiliar de la deliberación.

Un aspecto clave para entender la posición de Dewey al respecto es tener en consideración que las normas y los principios morales no son realidades fijas y permanentes a la espera de ser aplicadas. Dewey defiende una ética del agente en la que es este quien tiene la tarea siempre innovadora y creativa de encontrar la respuesta adecuada en la situación concreta. Dewey prolonga, en este sentido, la perspectiva aristotélica de que es el agente en situación quien, teniendo las disposiciones apropiadas, debe averiguar una respuesta que no está dada de antemano. "Como Aristóteles subrayó solo el buen hombre es un buen juez de lo que es verdaderamente bueno; se necesita un carácter adecuado y bien establecido para reaccionar inmediatamente con las correctas aprobaciones y condenas" (LW 7: 271). Cabe, en consecuencia, situar a Dewey en la línea de los teóricos de la virtud que han defendido que los hábitos excelentes tienen un papel epistémico, constitutivo respecto de la deliberación. Quiere esto decir que entienden que las virtudes son "el mejor criterio para determinar qué decisiones están justificadas" (Amaya, 2009, 33). Para Dewey, la disposición y hábitos del agente no son sólo una condición externa de posibilidad del ver y entender moral sino un elemento integral en la percepción y el razonamiento moral. Esto es, son las disposiciones las que permiten seleccionar aquellos aspectos de la situación que resultan relevantes y que pueden desempeñar el papel de constituir un motivo para la toma de decisión. Esta caracterización valdría para Dewey puesto que, en su interpretación, está claro que carácter y hábito condicionan de manera decisiva la selección de lo que es relevante. "Lo que un hombre ve o deja de ver con anticipación, lo que tiene en gran estima o en menosprecio, lo que considera importante o trivial, aquello a que se apega o que pasa por alto, lo que recuerda fácilmente u olvida de manera natural, son cosas que dependen de su carácter" (Dewey, 1964, 191). Así considerado, el hábito es en sí mismo un filtro que opera sobre la realidad seleccionando estímulos y señalando caminos del pensamiento. Además, el hábito no actúa sólo cuando propiamente se repite un acto, como sería usual en la manera de entenderlo, sino que pone de manifiesto su influencia sobre nuestra representación y comprensión del mundo justo cuando está en reposo. "Hábito quiere decir sensibilidad o accesibilidad especial a ciertas clases de estímulos, de predilecciones y aversiones permanentes; no simple repetición de 
actos específicos" (Dewey, 1964, 49). Así, la persona que tiene el hábito de andar tiene una percepción determinada de las distancias que es diferente del que tienen las personas con un hábito distinto. El hábito no indica únicamente algo pasivo en el ser humano sino que implica tanto un elemento de receptividad como de acción y transformación sobre el mundo.

Es esta presencia de la disposición del sujeto lo que servirá a Dewey para distanciarse de la manera en que los utilitaristas entienden la deliberación. La objetividad del cálculo utilitarista -la deliberación no es un cálculo algebraico, dice Dewey- parece dejar de lado que nuestras valoraciones están condicionadas por los estados del individuo. "Una persona de disposición bondadosa se siente lastimada ahora al pensar en un acto futuro que ocasione daño a los demás y, por lo tanto, está al acecho de consecuencias de esa clase, a las que concede gran importancia”. (Dewey, 1964, 191).

\section{La superación del dualismo entre lo interno y lo externo}

Sería, sin duda, un error interpretar que Dewey estimó que sólo había que fijarse en las condiciones subjetivas y no en las condiciones institucionales que, en buena medida, determinan los hábitos. Más aún, Naturaleza humana y conducta es, como su subtítulo indica -"una introducción a la psicología social"-, una obra que quiere poner de manifiesto la relevancia del medio social en la determinación de la conducta y un rechazo a la consideración de que la moral es un asunto interior. Puesto que, para Dewey, el hábito es tanto una función del medio como del propio organismo quiere llamar la atención sobre la importancia de modificar las condiciones sociales que conforman los hábitos y, con ello, los mecanismos de pensamiento. Estos no pueden explicarse por una lógica trascendente ni tampoco por cualquier tipo de determinismo naturalista que pretendiera explicarlos o justificarlos únicamente por referencia a una naturaleza humana dada. De manera reiterada, Dewey llamó la atención sobre el carácter tendencioso e ideológico de quienes apelaban a impulsos naturales como mecanismo de explicación de la realidad social y política. Se trate del egoísmo individual, de la agresión, de la buena voluntad, etc., en verdad siempre estamos ante la misma tesis: la presencia de componentes últimos invariables como justificación final del pensamiento y del comportamiento humano (Dewey, 1964, 105-122). La crítica a esta perspectiva subrayando los aspectos históricos, sociales y contextuales explica, en buena medida, la precedencia que Dewey concedió al hábito sobre el impulso. Aunque el impulso es primario en el sentido de ser un dato primitivo en la naturaleza humana, y el hábito es secundario en el sentido de ser adquirido, sin embargo, en la conducta del humano "lo adquirido es lo primitivo" (Dewey, 1964, 90). "El significado de las actividades innatas no es congénito sino adquirido" (Dewey, 1964,91). Y es que la experiencia humana está constituida por significados de manera que el impulso sólo adquiere sentido y significado a través del hábito que lo canaliza. Para Dewey, habitamos en un mundo de significados.

Puesto que el hábito es una función adaptativa del individuo al medio, la modificación de las circunstancias externas provoca, lógicamente, la transformación del hábito. No basta, por tanto, en moral con la apelación a la voluntad humana o con la invocación a factores ocultos o misteriosos. Dewey es explícito: “debemos actuar sobre el medio y no sólo sobre el corazón de los hombres" (Dewey, 1964, 32). Esta actuación sobre el medio ha de tener como base el estudio experimental de las condiciones bajo las que vivimos. No se trata de 
cambiar de modo caprichoso o aleatorio las condiciones bajo las que se forman hábitos, carácter y conducta. Las modificaciones han de ser resultado del análisis objetivo y consecuencial de las experiencias habidas. Para Dewey estas condiciones "pueden ser estudiadas tan objetivamente como las funciones fisiológicas, así como modificadas por medio de un cambio de elementos, personales o sociales". (Dewey, 1964, 27).

Ahora bien, si tan importante es el hábito para el proceso de deliberación $-y$, por ello, para el juicio moral y político-, y el hábito es un factor del medio tanto como del individuo, lo que podemos deducir es que la formación de este hábito, y con ello de la conducta y pensamiento del individuo, ha dejado de ser una cuestión privada y se ha convertido en una cuestión pública y política. La primacía concedida al concepto de hábito permite romper el dualismo ético/político, interior/exterior, y ello implica que los hábitos excelentes -o virtudes cívicas, visto desde la perspectiva política- son tanto un asunto individual como producto de las condiciones sociopolíticas. En la medida en que consideremos que la deliberación es una actividad central en democracia, parece lógico que, dada la relevancia de los hábitos para el pensamiento, deba haber un cierto grado de control en la formación de estos.

De ahí que, desde una perspectiva deweyana, los intentos de privatización de la formación de hábitos deban ser considerados como una pretensión antidemocrática. Esto permite una lectura crítica de una tendencia en auge en nuestra sociedad que se plasma en los crecientes intentos de privatización de los sistemas educativos, de comercialización de importantes aspectos de la socialización, de mercantilización de las formas de conocimiento, de control por parte de instituciones religiosas de la formación de creencias o, en definitiva, en la defensa de una concepción de la libertad entendida como ausencia de controles sociales. Esta posición de Dewey no tiene que suponer una defensa del control estatal de la formación del carácter sino, como desarrolló en Democracia y Educación, de un cierto grado de control social (Dewey, 1995, 34-40).

En todo caso, los mecanismos de deliberación individual son en buena medida la interiorización de los procedimientos externos de discusión. Caspary entiende que, para Dewey, la diferencia entre la deliberación pública y la deliberación particular es únicamente una cuestión de grado, siendo análogas en su funcionamiento e interactuando entre ellas (Caspary, 2000, 2014). A menudo se señala que la calidad del debate público depende estrictamente de la capacidad individual de tener en consideración otras perspectivas, ser sensibles a los hechos, estar correctamente informado, etc. Pero no tan frecuentemente se subraya el mecanismo opuesto que se deduce igualmente del planteamiento deweyano acerca de la deliberación; esto es, que la disminución de nuestra capacidad individual de deliberación puede ser fruto, en buena medida, de la disminución de la calidad del debate público. En este sentido, es una evidencia empírica que contextos más ricos desde el punto de vista intelectual fueron generadores de hábitos que propiciaron la investigación científica, artística o filosófica, de modo que la aparición simultánea de una cierta cantidad de grandes pensadores o científicos más que atestiguar una casualidad metafísica es fruto de un ambiente donde existía un caldo de cultivo apropiado. Pues bien, la degradación del diálogo y del debate en los espacios públicos aparece así como una amenaza creciente para el desarrollo de la individualidad.

En esta misma línea cabría resaltar que, puesto que la calidad de la deliberación depende de la capacidad de imaginar líneas de acción alternativas, diferentes, novedosas, entonces el cultivo de esta imaginación resulta crucial en el desarrollo de la inteligencia y de la demo- 
cracia. Recientemente, Nussbaum $(2001,2013)$ ha puesto de manifiesto la importancia del papel que la imaginación y la enseñanza de las humanidades desempeñan en la calidad de la democracia. Al mismo tiempo ha defendido que la democracia está en peligro por la progresiva mercantilización de los estudios que conlleva el arrinconamiento de las humanidades y, con ello, de la imaginación narrativa que constituye una de las capacidades fundamentales de la inteligencia. Se trata de una actualización de la tesis deweyana de que la democracia requiere una dimensión estética (música, literatura, filosofía, ...) que contribuya a la expansión de nuestra sensibilidad, nuestra capacidad para entender y considerar ideas desde diferentes perspectivas. Como el propio Dewey resumiera tomando la cita de Shelley "la imaginación es el gran instrumento del bien moral" (Dewey, 2008, 393)². En la deliberación deweyana la inteligencia ha de ser imaginativa para servir a los propósitos democráticos, y esta es producto de un hábito y una capacidad que ha de ser cultivada.

\section{Hábitos e instituciones}

El acento que Dewey puso en los hábitos y disposiciones ciudadanas ha llevado a considerar que el desdibujamiento de la fronteras entre lo interior y lo exterior, entre lo público y lo privado tiene como consecuencia una teoría política que se ciñe a las disposiciones ciudadanas como el elemento clave de la democracia. De hecho, diversos autores han echado en falta en la obra de Dewey referencias al aspecto institucional, lo han declarado "ingenuo" (Knight y Johnson, 1999, 566), o de acuerdo con el perfil que Bernstein realiza de la ética de Dewey "hay poco énfasis en el análisis de qué instituciones se requieren para el florecimiento de la democracia”. (Citado en Woods, 2014, 132).

Ahora bien, también es posible encontrar en Dewey textos que apoyan una interpretación diferente según la cual reconoció el papel de las instituciones en la configuración de la democracia. En este sentido es reveladora su posición en Liberalismo y acción social, donde señala que el uso social de la inteligencia permanecerá deficiente si todo lo que dice es que debe haber discusión y persuasión (1996, 104-105). Lo que en esta obra defiende es que la aplicación del uso de la inteligencia social implica planificación y control y, puesto que de lo que se trata es de modificar hábitos, las instituciones cumplen un papel fundamental en ello.

En este sentido, se ha ido abriendo paso una nueva línea de interpretación. Así, según Woods, "Dewey reconoció que el diseño institucional para dirigir la política y la economía fue y es necesario para permitir a la educación satisfacer su función social" (Woods, 2014, 134). Por su parte, Ralston (2010) ha defendido la compatibilidad entre pragmatismo e institucionalismo dado que, a su entender, la disputa en ciencia política entre institucionalismo y behaviorismo acerca del papel de las instituciones como el sujeto propio para el estudio normativo y empírico no hace sino reiterar un dualismo entre posiciones idealistas y antiidealistas que es justamente objeto de crítica desde la perspectiva de Dewey.

Más recientemente aún, se ha propuesto un nuevo modelo de democracia institucionalizada bajo el rótulo "experimentalismo democrático" que reivindica a Dewey como su

2 La relevancia de la imaginación para la democracia en la obra de Dewey es la tesis defendida por Thomas Alexander (1993, 1995). 


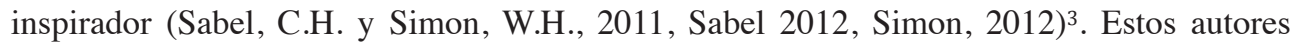
parten de reconocer que, efectivamente, Dewey consideró que "el cambio cultural sin cambio institucional sería inefectivo" (Simon, 2012). Sabel y Simon entienden como características propias del enfoque deweyano de las instituciones que estas deben: generar confianza por su capacidad para aprender y adaptarse a los continuos cambios y variaciones, ser descentralizadas, enfatizar el compromiso de cooperación entre los agentes y los afectados, y ser menos rígidas y más abiertas a la experimentación y a la evaluación de resultados. Se trata, desde la óptica experimentalista de Dewey, de extender el papel de la deliberación y la cooperación a todos los niveles de las instituciones y la administración.

Como Pappas ha reconocido, el experimentalismo democrático de Sabel y Simon supone una aportación al diseño de políticas institucionales desde la perspectiva del pragmatismo de Dewey; pero, al mismo tiempo, señala en la dirección que aquí queremos subrayar que no puede dejarse de lado que el éxito de las instituciones depende, en última instancia, de las virtudes ciudadanas. "Si el experimentalismo democrático no aborda la importancia de los hábitos y sus relaciones, su proyecto es susceptible de ser 'leve', 'formal', 'legalista' y 'vertical' a pesar de sus buenos objetivos e intenciones... El experimentalismo democrático no representa una oportunidad sin la encarnación de las virtudes cívicas". (Pappas, 2012, 70-71).

En este debate la toma en consideración de los hábitos como sede del ejercicio democrático pone en claro cuál es la situación de Dewey en relación con las instituciones. Si la tarea de la democracia es educativa, de conformación del tipo de subjetividad e individualidad democrática, sería ingenuo pensar que dicha tarea se puede acometer sin las instituciones adecuadas. De la misma manera que Dewey criticó a quienes reducían la democracia a su estructura formal, también se opuso a quienes realizan un interpretación moralista -en el sentido de no política-. Así, "la tarea educativa no puede quedar reducida a la esfera puramente mental, no puede prescindir de una acción que produzca cambios reales en las instituciones. Y una de las viejas pautas que han de ser alteradas es precisamente la idea de que las disposiciones y las actitudes pueden modificarse haciendo uso de medios 'morales', entendiendo por tales aquellos que actúan en el interior de las personas" (Dewey, 1996, 97)

Y es que las instituciones, siguiendo en esto a Ralston, consisten en creencias, hábitos y actividades o, en definitiva y como algunos teóricos lo denominan, en "una cultura organizacional" (Ralston, 2010, 75). Los hábitos hacen las instituciones del mismo modo que estas modelan de manera decisiva el carácter, la conducta y los hábitos de las personas ${ }^{4}$. Ya se ha señalado que, para Dewey, la verdadera tarea de la filosofía moral era la modificación de las condiciones del medio que hacían posible el desarrollo y crecimiento de los individuos. La cuestión, por consiguiente, no es que pensara que las instituciones son irrelevantes, sino que trató de enfatizar que no hay principios generales, ni instituciones fijas o permanentes que valgan como remedio por sí mismas para los males de la democracia. Entendió que era necesario llamar la atención sobre el hecho de que el medio y las condiciones que propician

3 El número 9 (2012) de Contemporary Pragmatism está dedicado al debate en torno a Dewey y el "experimentalismo democrático".

4 Sherman J. Clark defiende que hay hasta seis formas en que, conscientemente o inconscientemente, las leyes y la política moldean la conducta de las personas, lo que incluye aspectos como prohibir, exhortar, cultivar, conductas e ideas, así como proporcionar modelos de comportamiento y facilitar la discusión y el debate acerca de determinadas cuestiones. (Clark, 2013, 80-83). 
el desarrollo y la profundización en la democracia tienen que ser continuamente renovados para que cumplan su tarea de hacer viable el florecimiento de los individuos y la profundización en una sociedad democrática.

En todo caso, cabe admitir que Dewey se centró más en las disposiciones subjetivas que en las condiciones y transformaciones institucionales. En buena medida, porque dada la naturaleza contextual de su enfoque, una teorización de los mecanismos institucionales que pretendiera fijar las condiciones formales de la democracia habría sido contradictoria. Pero ello no significa que no le concediera importancia a la dimensión institucional y que no considerara que esta es una condición imprescindible para el desarrollo democrático. Ocurre que, estando en un país considerado formalmente democrático, Dewey entendió que los "individuos que son democráticos en pensamiento y acción son la garantía final para la existencia y perduración de instituciones democráticas" (LW 14: 92) y, de una manera que nos puede parecer ahora premonitoria, que el gran enemigo de la democracia no eran fuerzas externas sino problemas de legitimación, motivación, participación y convicción en la democracia por parte de los propios ciudadanos. Como el propio Dewey escribiera, "la democracia sólo tiene realidad por cuanto forma parte de la vida diaria" (Dewey, 1996, 204).

\section{El alcance normativo de los hábitos}

La importancia que Dewey concedió al condicionamiento social en la configuración de la individualidad, y la atención concedida en tal sentido al concepto de hábito, ha llevado a algunos a subrayar la conexión de la perspectiva de Dewey con la de algunos sociólogos, recientemente Bourdieu. Así, por ejemplo5, Colapietro señala: "la concepción de Bourdieu del habitus agranda y profundiza el rango de fenómenos conectados con la noción de hábito de Dewey" (Colapietro, 2004, 78). Esta ampliación del concepto de hábito de Dewey hace referencia a conceptos bourdesianos como los de capital (social, cultural, académico), campo y poder. Es cierto que, tanto en Bourdieu como en Dewey, el hábito se convierte en el conjunto de disposiciones que canalizan actitudes, modos de comportamiento y pensamientos. En el análisis de cómo los distintos campos de la actividad humana encierran mecanismos de organización del poder estructurados a través de los hábitos, los escritos de Bourdieu representan un paso adelante en relación con la perspectiva de Dewey haciéndolos más accesibles al estudio social empírico.

Ahora bien, al subrayar este camino de interpretación de Dewey, el del carácter social de la construcción de la individualidad, perdemos de vista otro lado de la cuestión que singulariza la posición de Dewey; esto es, la conexión de la idea de hábito con su propuesta de transformación social. Dicho de otro modo, se trata de la referencia normativa de los hábitos, su transformación en virtudes cívicas como medio de realización de una democracia moral.

Para Dewey, los hábitos no son exclusivamente mecanismos caprichosos de reproducción social o de reproducción de estructuras sociales o de jerarquías de poder, sino también respuesta a problemas y condiciones objetivas, modos de adaptación de los grupos sociales a los requerimientos que el medio plantea. Podemos así decir que, por un lado, ningún individuo

5 También Reich (2012): "la idea de hábito de Dewey es similar al más reciente concepto de habitus desarrollado por el sociólogo francés Pierre Bourdieu”. En Garrison, 2012, 5. 
ni ninguna generación está preparada para responder ante el mundo de una manera que esté más allá de los hábitos adquiridos (un carácter y talante radicalmente igualitario fue imposible en sociedades esclavistas, o un espíritu liberal en una sociedad jerárquica, estamental o cerrada). Pero, por otro, los hábitos, en tanto que permanecen estancados y no evolucionan preparando a las personas para responder de manera más inteligente y permitiendo una mejor adaptación a las circunstancias del medio, se convierten en un obstáculo para la inteligencia moral. "El hábito no excluye el uso del pensamiento, pero determina los canales a través de los que opera" (LW 2: 335). Por tanto, los hábitos, además de establecer límites, son también las condiciones de posibilidad del uso de una inteligencia abierta y experimental. De manera que no se trata de subrayar sólo el condicionamiento de la inteligencia por parte de los hábitos, sino también la capacidad de aquella de reobrar sobre estos. En Dewey los hábitos tienen una dimensión teleológica. No son simplemente actividades que se repiten serialmente, sino que están estructuradas hacia la resolución del curso de acción. No son estructuras pasivas, sino que persiguen el incremento de significado de la acción ${ }^{6}$. En este sentido, y de acuerdo con la interpretación de Dewey de que la tarea de la ética es la aplicación de la inteligencia a la evaluación moral, podemos decir que no todos los hábitos son moralmente iguales. Los hábitos que preparan y conducen para la acción inteligente, y moralmente excelente, son las virtudes, y Dewey no distingue entre virtudes epistémicas y morales ${ }^{7}$. La vinculación que establece entre ética y ciencia se aclara en el terreno de los hábitos. Quienes interpretaron a Dewey de manera positivista erraron claramente al considerarlo un reduccionista puesto que lo que estaba defendiendo era la generalización de los hábitos que son propios del quehacer científico. Es, por tanto, una llamada de atención al hecho de que no podemos avanzar en cuestiones morales y políticas si no es por una extensión a la ciudadanía de las capacidades de autorreflexión, de juzgar y evaluar en función de los hechos y las consecuencias, de contrastar información, etc... Se trata de la extensión social de lo que Dewey definió como el método de la investigación.

Es en Ethics (LW 7) donde Dewey manifiesta con mayor claridad el carácter normativo de los hábitos y el papel de la inteligencia en su reconstrucción. Así, distingue entre la "moralidad convencional o de costumbres" y la "moralidad reflexiva". Esta última "identifica la virtud no con lo que de hecho es aprobado sino con lo que debería ser aprobado" (LW 7: 253). Desde luego esto implica reconocer la importancia de la tarea de la reflexión en su esfuerzo por mediar bienes y normas y dar respuesta a las demandas de cada situación. Esta tarea es posible porque los hábitos hacen referencia a bienes objetivos sin los que los hábitos no pasarían a ser virtudes, quedándose en el ámbito de la moralidad de costumbres.

Así pues, percepción, creatividad, atención a los otros, sensibilidad, son rasgos de la acción inteligente. Pues bien, hay hábitos que favorecen estos rasgos y preparan en mayor medida para la atención a los otros, el análisis empírico de los hechos, el diálogo y la toma en consideración de los otros, para la reflexión y la deliberación. La virtud de la tolerancia capacita al individuo para tener en consideración la legitimidad de las diferencias; la virtud de la solidaridad y la generosidad capacita al individuo para un mayor sentido de la igualdad y un rechazo a la desigualdad arbitraria e injusta; el espíritu de lealtad tanto a las institu-

6 Sobre la estructura teleológica de los hábitos, veáse Alexander (1993, 385).

7 Pappas defiende dicha interpretación contra las que han realizado Talisse y Misak (Pappas, 2008, 255). 
ciones democráticas como a las personas capacita a los individuos para un trato decente e imparcial con los demás; la virtud del espíritu crítico prepara para el examen detenido de ideas y principios a la luz de la consecuencias; la virtud del esfuerzo nos capacita para el rechazo de una vida carente de sentido del logro y del afán de mejora. Estas virtudes, estos hábitos que preparan para la acción moral excelente, son el núcleo alrededor del cual gira el significado de la democracia entendida como modo de vida.

En resumen, tomar en consideración la perspectiva que Dewey nos dejó sobre los hábitos supone no sólo situarla en la línea de los sociólogos que han subrayado el condicionamiento social del pensamiento, sino también junto a aquellos que, aun reconociendo dicho influjo social, han entendido que el ser humano es un ser abierto a la experiencia, capaz de aprender de ella y de transformar la acción haciéndola más armónica en relación con el medio y con los otros seres humanos. Lo distintivo en este punto es que son los hábitos los que abren una dimensión normativa que permite juzgar actitudes e instituciones.

\section{La primacía de los hábitos y la filosofía política de Dewey}

Dewey entendió que la democracia es un modo de vida caracterizado por la investigación social cooperativa (Honneth, 1998). Es en este contexto de la inteligencia cooperativa como debemos entender su caracterización de la deliberación. Guiado por la idea de comunidad de investigación Dewey entendió que la democracia radicaba en la cooperación social guiada por el uso y método de la inteligencia y, por consiguiente, que la deliberación había de ser un elemento integral suyo.

Autores como Misak (2000) y Talisse $(2005,2007)$ han argumentado a favor de una interpretación política del pragmatismo, si bien basándose en la obra de Peirce, de acuerdo con la cual lo singular de esta perspectiva filosófica reside en una renovada defensa epistémica de la democracia bajo la convicción de que esta guarda relación con la manera en que defendemos y justificamos nuestras creencias. La consecuencia de su perspectiva es que la aceptación del marco epistemológico del pragmatismo conduce a la defensa de una concepción deliberativa de la democracia en la que lo relevante para la mejora de la calidad de la democracia radica en un cierto perfeccionismo epistémico, en la mejora de las capacidades argumentativas y deliberativas de la ciudadanía (Talisse, 2005, 118-121, 2007, 85-98).

Frente a autores como Posner (2003), y en diferente modo también el propio Rorty (1991), que han insistido en que la inspiración pragmatista supone el abandono de las pretensiones epistémicas de la democracia, cabe afirmar -de acuerdo con Talisse o Misak- que la concepción deliberativa de la democracia es la que se adecua más a la inspiración filosófica que representa el pragmatismo. Así, para los defensores de la concepción deliberativa, en democracia la actividad política ha de girar en torno a ofrecer y aceptar argumentos, bajo la idea de que las decisiones correctas son aquellas que son aceptadas por buenas razones, que estas emergen en el intercambio de ideas y que la mayor parte de los ciudadanos pueden o podrían aceptar.

Ahora bien, estas interpretaciones no dan cuenta del verdadero giro que en las teorías deliberativas propone la obra de Dewey. El modelo deliberativo de democracia puede ser acusado de tener un sesgo excesivamente racionalista, de atender exclusivamente a los elementos cognitivos que intervienen en la elaboración del juicio práctico político. Desde la 
perspectiva pragmatista se podrá compartir con los teóricos de la deliberación el rechazo a otras formas de entender la democracia (agregativa, procedimentalista, pluralista, minimalista,..) pero, por otro lado, y de acuerdo con la perspectiva desarrollada por Dewey que hace de los hábitos el eje de su concepción normativa, se debe acusar a las teorías deliberativas de no atender suficientemente a los aspectos no estrictamente cognitivos que son parte consustancial del proceso práctico de elaboración del juicio, de dar primacía al componente epistémico de la democracia frente a su caracterización como empresa ética. En este sentido autores como Pappas (2008), Shalin (2011) o Stout (2005) habrían venido a acentuar que el enfoque deweyano y pragmatista supone subrayar que otros componentes no epistémicos son determinantes de la democracia.

En esta dirección, J. Bohman ha situado el sentido de la deliberación en el terreno pragmático al señalar, de un lado, el carácter contextual de la deliberación y, de otro, que el éxito de esta consiste no tanto en un acuerdo racional como en mantener y reforzar los mecanismos cooperativos. Refiriéndose a la deliberación, señala: "Comienza con una situación problemática en la que la coordinación se ha interrumpido. Triunfa cuando los actores son capaces de cooperar de nuevo... El éxito se mide no por el fuerte requisito de que todos estén de acuerdo con el resultado sino con el más débil de que los agentes estén suficientemente convencidos de su continua cooperación. Un resultado de una decisión actual es aceptable cuando las razones detrás de ella son suficientes para motivar la cooperación de todos aquellos que deliberan" (Bohman, 1996, 33).

Bohman prolonga la línea abierta por Dewey al indicar que lo que ha de guiar la deliberación es la voluntad de continuar la cooperación y, por tanto, que son elementos actitudinales los que modelan el proceso de deliberación. En esta interpretación, las ideas se convierten en un instrumento al servicio del entendimiento y la cooperación. Ahora bien, si esta interpretación de Bohman es adecuada como respuesta a una consideración teórica e intelectualista de la deliberación, resulta insuficiente desde la perspectiva de los hábitos señalados con anterioridad. Lo que no indica Bohman es que esa voluntad de cooperación depende, en definitiva, del desarrollo de capacidades y disposiciones sin las que esa cooperación es posible. De hecho, resalta la importancia de ponerse en el papel del otro -recurriendo a Mead-como uno de los mecanismos importantes de la deliberación. Desde luego, se trata de una de las capacidades y actitudes que exige una voluntad de cooperación democrática. Pero también es necesario tener en consideración que hay otros hábitos que hacen posibles actitudes y disposiciones que son igualmente definitorias de lo democrático; por ejemplo, las que hacen referencia a la inclusividad y la integración, el aprecio por la libertad, y la sensibilidad hacia la igualdad. Son estos hábitos los que, diseminados entre la ciudadanía, son los garantes de la existencia del buen funcionamiento de los mecanismos deliberativos que harán posible la resolución democrática de los conflictos. El ensanchamiento y extensión de los hábitos, de las actitudes y disposiciones cívicas, deviene un elemento esencial para la mejora de la calidad de la democracia. Siguiendo en esto a Pappas (2008) el giro deliberativo de la teoría democrática debe ser completado a su vez con un giro pragmático en el sentido marcado por J. Dewey. "Por esto para Dewey el giro deliberativo debe ser más que un giro epistémico. La democracia es mucho más que epistemología. Hay más en la investigación democrática que el intercambio de razones y argumentos por pensadores con excelentes hábitos epistémicos" (Pappas, 2008, 255). 
La inversión de la lectura de las prácticas deliberativas que el pragmatismo exige consiste en interpretar que no es que el hábito o la virtud cívica sea un instrumento necesario para la práctica deliberativa, como parte de las teorías deliberativas y, especialmente, republicanas habrían venido a afirmar sino a la inversa, que la prueba de fuego de la práctica deliberativa radica en su capacidad para contribuir a la mejora de los individuos. Habría que juzgar, siguiendo la máxima pragmática, el valor de las distintas maneras de entender la democracia por el tipo de ciudadano que contribuye a crear, por el tipo de hábitos que contribuye a diseminar entre los ciudadanos. "La democracia tiene muchos significados, pero si tiene un significado moral, lo encontraremos en que establece que la prueba suprema de todas las instituciones políticas y de todos los dispositivos de la industria está en la contribución de cada una de ellas al desarrollo acabado de cada uno de los miembros de la sociedad." (MW 12:186). El valor de la deliberación estriba, por consiguiente, en que nos hace autónomos, sensibles a los argumentos, establece lazos entre los individuos contribuyendo a la formación de una personalidad más rica, nos hace más libres e iguales, y nos vincula a las normas. Por el contrario, otras teorías de la democracia, como en general las teorías minimalistas, desincentivan la responsabilidad social de los individuos, animan a que los ciudadanos se consideren competidores y no colaboradores, y terminan por dibujar una sociedad donde triunfa la manipulación y el ansia de poder.

En este punto el elemento a tener en consideración es que el juicio, además de normativamente orientado desde los requerimientos de la coordinación social, debe estarlo también hacia la consecución de bienes que consideramos esenciales desde la perspectiva de la democracia. La democracia no es, vista de este modo, un sistema de organización política y social desprovisto de valores morales. No es, como tantos liberales han defendido, un sistema neutro que permite la más amplia variedad posible de posiciones morales comprehensivas. Antes al contrario, caracteriza a la democracia el haber configurado un marco de valores que aspiramos a convertir en realidad y que se encuentran identificados en declaraciones internacionales y, a menudo, en las propias constituciones. Stout (2005) ha reivindicado, desde la óptica del pragmatismo, que la democracia es una tradición, que no es exclusivamente un asunto de dar y recibir razones sino una forma de vida que incorpora actitudes, hábitos, disposiciones y motivaciones que implican una sensibilidad hacia un conjunto de bienes que son identificables en el seno de la tradición democrática. La idea es que "la democracia se genera a través de prácticas sociales que incluyen hábitos, actitudes y disposiciones en sus participantes" (Stout, 2005, 203). La democracia no es un logro intelectual que se impone frente a costumbres y hábitos, sino una práctica que contiene el elemento reflexivo entre sus más destacados caracteres, que se autocorrige en el proceso mismo del movimiento y que se orienta a la consecución de bienes e intereses que compartimos.

\section{Conclusión}

La primacía de los hábitos en la explicación del comportamiento humano y su relevancia en el proceso de deliberación nos dan algunas claves para situar el pensamiento político de Dewey en el marco de las teorías de la democracia.

Si bien Dewey puede ser considerado un defensor de la democracia deliberativa, esto es, de la democracia entendida como la búsqueda inteligente y cooperativa de las soluciones a nuestros problemas, la importancia que concede a los elementos no cognitivos le aleja de 
otros teóricos de la democracia deliberativa. No se trata de negar que la democracia ha de ser el régimen donde primen las mejores razones, sino poner de manifiesto que las razones son siempre situadas y en sujetos encarnados. La primacía que Dewey otorgó a los hábitos en el proceso de deliberación tiene como consecuencia el alejamiento de una interpretación idealista del intercambio de ideas. En democracia no queremos argumentos y razones en sí y por sí, sino aquellos dirigidos hacia la realización de determinados valores, los guiados por ciertos deseos y pasiones, los que permiten y ayudan a la continuidad de la cooperación en la resolución de los problemas.

Es la presencia de los hábitos en la deliberación lo que hace a Dewey sostener que la transformación social lo que requiere es, básicamente, educación. De lo que se trata es de modificar los hábitos para conseguir que la democracia se convierta en un modo de vida, de promover las condiciones sociales que hacen posible la existencia de ciudadanos virtuosos. Sea cual sea el problema político que se trate lo que necesitamos, antes que una teoría política que nos indique lo correcto, es una ciudadanía que pueda interpretar la información a la luz de los principios democráticos, que se atenga a la evidencia de los hechos y evalúe las consecuencias que se siguen de ellos. Necesitamos ciudadanos orientados hace la consecución de los bienes democráticos, instituciones sociales y políticas que tengan un carácter educativo, y un sistema educativo que logre ciudadanos virtuosos.

La consecuencia de la interpretación de Dewey del peso de los hábitos en la deliberación se traduce en una teoría política que consiste en una propuesta normativa de la educación moral del ciudadano. Ello implica también una desconfianza en los cambios sociales radicales, y el rechazo de la convicción de que basta con las modificaciones institucionales para transformar la sociedad. Dewey no fue un revolucionario sino un meliorista convencido tanto de las posibilidades de transformación social como de que dicha transformación sólo podía hacerse modificando los hábitos existentes. En consecuencia, la tarea de la moral no puede ser otra que el cultivo de los mejores hábitos, el desarrollo de nuestras disposiciones y capacidades.

En definitiva, el antiintelectualismo desplegado por el pragmatismo en epistemología encuentra su paralelo en el ámbito de la teoría deliberativa al subrayar la importancia de los hábitos y de la virtud cívica para la resolución de los problemas democráticos. La necesidad de contar con políticas e instituciones educativas es una implicación lógica de la relevancia de la virtud ciudadana en teoría política, para la que la realización de lo bueno y lo justo depende del cultivo de la capacidad de percepción de esas virtudes y de las habilidades racionales que incorporan pretensiones universalizadoras. Como señala Shalin, "la democracia es más que un discurso, una cultura cívica" $(2011,477)$. Es por ello por lo que la democracia debe contener no sólo un logos sino también un pathos y un ethos dejando espacio no sólo para el progreso intelectual sino también para la creatividad emocional y la imaginación moral (Shalin, 2011, 478).

Quizás valga como diagnóstico y receta para enfrentar los difíciles momentos que atraviesa la política y la democracia, tanto en España como en Europa la siguiente cita de Dewey: "Lo moral es desarrollar el discernimiento, la capacidad para juzgar el sentido de lo que estamos haciendo y para usar ese juicio en la orientación de lo que hacemos, no por medio del cultivo directo de algo llamado conciencia, razón o facultad de conocimiento moral, sino fomentando aquellos impulsos y hábitos que sabemos que nos hacen sensibles, generosos, imaginativos e imparciales para percibir la tendencia de nuestras actividades incipientes" (Dewey, 1964, 193). 


\section{Bibliografía}

Alexander, T. (1993), "John Dewey and the Moral Imagination: Beyond Putnam and Rorty toward a Postmodern Ethics", Transactions of the Charles S. Peirce Society, (Indiana) 29.3 (Summer), pp. 369-400.

Alexander, T. (1995), "John Dewey and the Roots of the Democratic Imagination". Albany SUNY, 131-154, en Recovering Pragmatists Voice: The Classical Tradition, Rorty and the Philosophy of Communication, ed. By Leonor Langsdorf, New York, Albany State University of New York Press.

Amaya, A. (2009), Virtudes judiciales y argumentación: una aproximación a la ética jurídica. México. Tribunal Electoral del Poder Judicial de la Federación.

Amaya, A., Hock Lai, H., (eds) (2013), Law, Virtue and Justice, Oxford, Hart Publishing.

Bohman, J. (1996), Public Deliberation. Pluralism, Complexity and Democracy, Cambridge, Massachussetts Institute of Technology Press.

Caspary, W. R. (2000), Dewey on Democracy, New York, Cornell University.

Clark, S. J. (2013), “Neoclassical Public Virtues” en Amaya, A. y Hock Lai, Ho, (eds) (2013).

Colapietro, V. (2004), "Doing -and Undoing- the Done Thing: Dewey and Bourdieu on Habituation, Agency, and Transformation". Contemporary Pragmatism. Vol 1, № 2 , 65-93.

Dewey, J. (1969-1991), The Collected Works of John Dewey, Carbondale, Southern Illinois University Press.

Dewey, J. (1964), Naturaleza humana y conducta, México, Fondo de Cultura Económica.

Dewey, J. (1995), Democracia y educación, Madrid, Ed. Morata.

Dewey, J. (1996), Liberalismo y acción social, Valencia, Edicions Alfons El Magnánim, Generalitat Valenciana.

Dewey, J. (2008), El arte como experiencia, Barcelona, Paidós.

Fesmire, St. (2003), John Dewey and Moral Imagination: Pragmatism in Ethics, Bloomington, Indiana University Press.

Honneth, A. y Farrell J. M. (1998), "Democracy as Reflexive Cooperation: John Dewey and the Theory of Democracy Today". Political Theory. Vol. 26, No. 6 (Dec., 1998), pp. 763-783.

Garrison, J., Neubert, S. Reich, K. (2012), John Dewey's Philosophy of Education: An Introduction and Recontextualization for Our Times, New York, Palgrave MacMillan.

Green, Judith M. (2014), R. Bernstein and the Pragmatic Turn in Contemporary Philosophy, New York, Palgrave MacMillan.

Knight, J., and Johnson, J. (1999), "Inquiry into Democracy: What Might a Pragmatist Make of Rational Choice Theories? American Journal of Political Science, 43, pp. 566-589.

Misak, Ch. (2000), Truth, Politics, Morality: Pragmatism and Deliberation, London, Routledge.

Nussbaum, M. (2001), El cultivo de la humanidad. Barcelona, Ed. Andrés Bello.

Nussbaum, M. (2013), Sin ánimo de lucro. Por qué la democracia necesita de las humanidades. Oviedo, Katz Editores.

Pappas, G. F. (2008) John Dewey's Ethics. Democracy as Experience, Bloomington, Indiana University Press. 
Pappas, G.F. (2012) "What would John Dewey say about Deliberative Democracy and Democratic Experimentalism?”, Contemporary Pragmatism, Vol. 9 No 2, (December), pp. 57-74.

Ralston, S. J. (2010), “Can Pragmatists be Institutionalists? John Dewey Joins the Non-ideal/ Ideal Theory Debate" Human Studies 33, pp. 65-84.

Rorty, R. (1991), Contingencia, ironía y solidaridad, Paidós Básica, Barcelona.

Sabel, Ch. F. y Simon, W.H. (2011), "Minimalism and Experimentalism in the Administrative State”, Georgetown Law Journal, vol. 100, № 1, November 2011.

Sabel, Ch. (2012) "Dewey, Democracy and Democratic Experimentalism" Contemporary Pragmatism, Vol. 9 No 2 (December), pp. 35-55

Shalin, Dimitri N., (2011) Pragmatism and Democracy. New Brunswick, Transactions Publisher.

Simon, W. H. (2012) "The Institutional Configuration of Deweyan Democracy", Contemporary Pragmatism, Vol. 9 No 2 (December), 5-34.

Stout, J. (2005), Democracy and Tradition, New Jersey, Princeton University Press.

Talisse, Robert B. (2005) Democracy after Liberalism: Pragmatism and Deliberative Politics. New York, Routledge.

Talisse, R. B. (2007), A Pragmatist Philosophy of Democracy, New York, Routledge.

Woods, David W. (2014), "Democratic Community Participation: Bernstein between Dewey and an Achieved Deeply Democratic Future" en Green, J. (2014) pp. 131-146. 